\title{
BMJ Open Cow's Milk Fat Obesity pRevention Trial (CoMFORT): a primary care embedded randomised controlled trial protocol to determine the effect of cow's milk fat on child adiposity
}

Shelley M Vanderhout, ${ }^{1,2,3}$ Mary Aglipay, ${ }^{2}$ Catherine Birken, ${ }^{1,4,5,6}$ Patricia Li, ${ }^{7}$ Deborah L O'Connor, ${ }^{1}$ Kevin Thorpe, ${ }^{3,5}$ Evelyn Constantin, ${ }^{8}$ Marie-Adele Davis, ${ }^{9}$ Mark Feldman, ${ }^{4,6}$ Geoff D C Ball, ${ }^{10}$ Magdalena Janus (D) , ${ }^{11}$ Peter Jüni, ${ }^{3,5}$ Anne Junker, ${ }^{12}$ Andreas Laupacis, ${ }^{3,5}$ Mary L'Abbé, ${ }^{1}$ Heather Manson, ${ }^{13}$ Myla E Moretti, ${ }^{14}$ Nav Persaud (D) , ${ }^{3,15}$ Jessica A Omand (D) , ${ }^{4}$ Clare Relton, ${ }^{16}$ Peter Wong, ${ }^{4,6}$ Hirotaka Yamashiro, ${ }^{17}$ Erika Tavares, ${ }^{18}$ Shannon Weir, ${ }^{18}$ Jonathon L Maguire (D) ${ }^{1,2,3,5}$

To cite: Vanderhout SM, Aglipay M, Birken C, et al. Cow's Milk Fat Obesity pRevention Trial (CoMFORT): a primary care embedded randomised controlled trial protocol to determine the effect of cow's milk fat on child adiposity. BMJ Open 2020;10:e035241. doi:10.1136/ bmjopen-2019-035241

- Prepublication history and additional material for this paper are available online. To view these files, please visit the journal online (http://dx.doi. org/10.1136/bmjopen-2019035241).

Received 24 October 2019 Revised 13 March 2020 Accepted 09 April 2020

Check for updates

(C) Author(s) (or their employer(s)) 2020. Re-use permitted under CC BY-NC. No commercial re-use. See rights and permissions. Published by BMJ.

For numbered affiliations see end of article.

Correspondence to Dr Jonathon L Maguire; jonathon.maguire@utoronto.ca

\section{ABSTRACT}

Introduction Cow's milk is a dietary staple for children in North America. Though clinical guidelines suggest children transition from whole ( $3.25 \%$ fat) milk to reduced $(1 \%$ or $2 \%)$ fat milk at age 2 years, recent epidemiological evidence supports a link between whole milk consumption and lower adiposity in children. The purpose of this trial is to determine which milk fat recommendation minimises excess adiposity and optimises child nutrition and growth.

Methods and analysis Cow's Milk Fat Obesity pRevention Trial will be a pragmatic, superiority, parallel group randomised controlled trial involving children receiving routine healthcare aged 2 to $4-5$ years who are participating in the TARGet Kids! practice-based research network in Toronto, Canada. Children $(n=534)$ will be randomised to receive one of two interventions: (1) a recommendation to consume whole milk or (2) a recommendation to consume reduced (1\%) fat milk. The primary outcome is adiposity measured by body mass index z-score and waist circumference z-score; secondary outcomes will be cognitive development (using the Ages and Stages Questionnaire), vitamin D stores, cardiometabolic health (glucose, high-sensitivity C-reactive protein, non-high density lipoprotein (nonHDL), low density lipoprotein (LDL), triglyceride, HDL and total cholesterol, insulin and diastolic and systolic blood pressure), sugary beverage and total energy intake (measured by 24 hours dietary recall) and cost effectiveness. Outcomes will be measured 24 months postrandomisation and compared using analysis of covariance (ANCOVA), adjusting for baseline measures. Ethics and dissemination Ethics approval has been obtained from Unity Health Toronto and The Hospital for Sick Children. Results will be presented locally, nationally and internationally and published in a peer-reviewed journal. The findings may be helpful to nutrition guidelines for children in effort to reduce childhood obesity using

\section{Strengths and limitations of this study}

- Cow's Milk Fat Obesity pRevention Trial (CoMFORT) addresses a common and clinically important problem through the evaluation of two nutritional interventions in primary healthcare.

- Nesting CoMFORT within the TARGet Kids! prospective cohort study may improve follow-up and allow future study of intervention effects later in childhood.

- Using a patient-centred proportional consent model may enhance inclusion of under-represented groups and increase study efficiency.

- While the pragmatic intervention is a physician recommendation, adherence to the recommended milk fat content may be variable.

a simple, inexpensive and scalable cow's milk fat intervention.

Trial registration number NCT03914807; pre-results.

\section{BACKGROUND}

Inexpensive and widely accessible cow's milk has been a dietary staple for children in North America for over a century. The majority of North American children consume cow's milk on a daily basis. ${ }^{12}$ Cow's milk provides children with nutrients for growth and development such as protein, carbohydrates, calcium, vitamins $\mathrm{A}$ and $\mathrm{D}$ and fat. The National Health Service, ${ }^{3}$ Canadian Paediatric Society ${ }^{4}$ and the American Academy of Pediatrics ${ }^{5}$ recommend whole $(3.25 \%$ fat $)$ cow's milk for children beginning at 1 year of age to support optimal development in a 
period of rapid growth and high energy demand. ${ }^{6}$ In an effort to curb childhood obesity, children are recommended to transition from whole to reduced fat $(1 \%$ or $2 \%$ ) cow's milk starting at 2 years of age. ${ }^{3-5}$ However, this recommendation is based on low quality evidence (Grading of Recommendations Assessment, Development and Evaluation (GRADE) ${ }^{8}$ score of $\left.0-1\right)^{9}$ derived primarily from consensus opinion. ${ }^{910}$

It is unclear whether switching from whole milk to reduced fat milk at age 2 years is beneficial. Observational evidence supports that children who consume whole milk have a lower risk of overweight or obesity relative to children who consume reduced $(0.1 \%-2 \%)$ fat milk. ${ }^{11-13}$ A systematic review and meta-analysis of observational evidence for the relationship between cow's milk fat and child adiposity reported that children who consumed whole milk had 1/3 lower odds of overweight and obesity compared with children who consumed reduced $(0.1 \%-$ $2 \%)$ fat milk. ${ }^{14}$ Proposed mechanisms include higher satiety offered by whole milk due to hormonal responses to dietary milk fat consumption ${ }^{13} 15$ thus displacing nutrient-poor foods or sugary beverages. Another theory is that a low fat, high protein diet in early childhood may programme a 'thrifty metabolism', where the body adapts by storing excess energy as fat. ${ }^{16}$ Conversely, a higher fat diet may metabolically programme higher energy utilisation and lower energy storage. ${ }^{16}{ }^{17}$ Fatty acids found in cow's milk, such as trans-palmitoleic acid and conjugated linoleic acid, may be protective against excess adiposity. ${ }^{18} 19$ Reverse causality could also explain this relationship, where parents may choose milk with a fat content to counter-balance the adiposity of their child (ie, higher fat milk for a leaner child) ${ }^{20}$ Given the financial burden of overweight and obesity on healthcare systems worldwide, ${ }^{21}$ determining which milk fat recommendation in childhood is effective in lowering a child's risk of developing excess adiposity, may result in substantial healthcare savings in the future.

It is possible that cow's milk fat may also result in other beneficial health effects. Higher circulating levels of transpalmitoleic acid have been associated with lower adiposity, low density lipoprotein (LDL) cholesterol, insulin resistance and triglycerides, and positively associated with high density lipoprotein (HDL) cholesterol, in several large adult cohort studies. ${ }^{18}$ During early childhood, dietary fat consumption is known to support cognitive development, which usually concludes around 6 years of age. ${ }^{22}$ Cow's milk fat may promote brain development due to its essential fatty acid content (eg, linoleic acid) which may manifest in gains across multiple developmental domains including social, emotional and physical. ${ }^{23}{ }^{24}$ The ratio of essential fatty acids linoleic to alpha-linoleic acid (n-6 to n-3) in whole cow's milk is believed to optimise circulating docosahexaeonic acid, ${ }^{25}$ which is an important fatty acid to brain growth and function. ${ }^{26}$

Parents and clinicians have expressed interest in evidence-based guidelines for milk fat during early childhood, but rely on different nutrition information sources including primary healthcare recommendations. ${ }^{27}$ Both whole and reduced fat milk are currently recommended to families with young children receiving primary healthcare. ${ }^{27}$ To inform clinical practice and evidence-based guidelines, randomised controlled trial evidence is needed to determine whether switching to reduced fat milk at 2 years of age or continuing with whole milk beyond 2 years of age results in improved growth, cardiovascular and cognitive developmental outcomes.

\section{OBJECTIVES}

\section{Overall objective}

To determine whether a primary healthcare recommendation for whole $(3.25 \%$ fat $)$ versus reduced fat $(1 \%$ fat) milk in early childhood can: (1) reduce adiposity; (2) improve cardiovascular health; (3) improve cognitive development; (4) increase vitamin D stores and (5) reduce sugary beverage consumption and total energy intake at 24 months postrandomisation.

\section{Hypotheses}

We hypothesise that recommending whole milk between 1 and 4-5 years of age versus transitioning to reduced fat milk at 2 years will result in the following outcomes at 24 months postrandomisation:

- Primary outcome: lower excess adiposity measured by: (1) body mass index z-score (zBMI) and (b) waist circumference z-score (zWC).

- Secondary outcome 1: lower risk of cardiovascular disease measured by: blood pressure, non-HDL, LDL, triglyceride, HDL and total cholesterol, glucose, insulin, high-sensitivity C-reactive protein (hsCRP) and glycosylated haemoglobin.

- Secondary outcome 2: higher vitamin D status measured by serum 25 -hydroxyvitamin $\mathrm{D}(25(\mathrm{OH}) \mathrm{D})$.

- Secondary outcome 3: better cognitive developmental scores measured by the Ages and Stages Questionnaire (ASQ) and the Early Development Instrument (EDI).

- Secondary outcome 4: lower sugary beverage consumption and total energy intake measured by the Automated Self-Administered 24 hours Recall tool (ASA-24).

- Secondary outcome 5: lower financial costs to both families and the healthcare system.

\section{STUDY DESIGN AND METHODS}

This will be a pragmatic, parallel group, superiority, randomised controlled trial. The study will include two active arms: (1) primary healthcare recommendation to consume whole milk starting at 2 years of age and (2) primary healthcare recommendation to consume reduced fat $(1 \%)$ milk starting at 2 years of age. This protocol has been designed following the 2013 Standard Protocol Items: Recommendations for Interventional Trials guide$\operatorname{lines}^{28}$ and registered at clinicaltrials.gov. Trial results will 


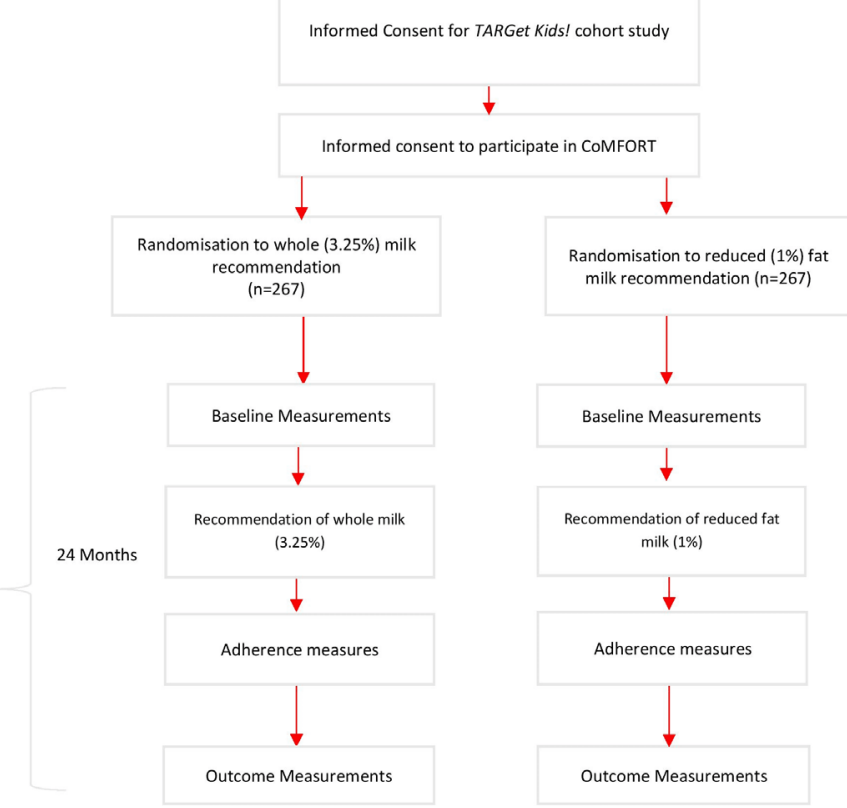

Figure 1 Trial flow diagram. CoMFORT, Cow's Milk Fat Obesity pRevention Trial.

be reported according to the Consolidated Standards of Reporting Trials guidelines for pragmatic trials. ${ }^{29}$

\section{Study setting}

Healthy children aged 1.5-2.99 years will be recruited during a routine well-child doctor's visit at 12 participating TARGet Kids! academic paediatric or family medicine group practices in Toronto and Montreal over 2 years. The TARGet Kids! primary care research network and children's longitudinal cohort study is a collaboration between academic health outcome researchers at the University of Toronto, McGill University and a network of over 100 university affiliated primary healthcare providers (www.targetkids.ca). ${ }^{30}$ Children participating in TARGet Kids! provide anthropometric, lifestyle and developmental information and a blood sample at routine wellchild visits. Recruitment started in February 2020 and is expected to take 24 months to complete enrolment.

\section{Inclusion criteria}

Children who are: (1) healthy by parental report (characterised as not living with chronic or acute illness, except for asthma); (2) 1.5-2.99 years of age; (3) involved in a TARGet Kids! academic paediatric or family medicine group; (4) are from families with verbal communication in English or French.

\section{Exclusion criteria}

Children who: (1) have Prader-Willi syndrome or other syndrome associated with obesity; (2) have severe developmental delay which impacts daily functioning; (3) are considered failure to thrive (children with zBMI values $\leq-2$ are unlikely to benefit from obesity prevention); (4) are siblings of trial participants as families may share milk or (5) do not consume cow's milk by choice, lactose intolerance or allergy.

\section{Interventions}

During a scheduled well-child visit, children aged 1.5-2.99 years will be randomised to one of two interventions currently provided in primary care ${ }^{27}$ : (1) whole fat milk recommendation and (2) reduced fat milk recommendation (figure 1). Standardised training sessions based on current clinical guidelines will be provided to participating primary care providers with quarterly reminders to ensure consistency in the provided recommendations. For children randomised to either group, research assistants will notify the child's physician of their allocated recommendation immediately prior to the clinical encounter. All participating healthcare providers have provided consent to participate in the randomisation process. Intervention scripts for physicians can be found in the online supplementary file.

\section{Whole milk recommendation}

Children randomised to the whole milk recommendation will receive a primary care recommendation to consume $500 \mathrm{~mL}$ of whole fat $(3.25 \%)$ milk per day instead of transitioning to reduced fat $(1 \%)$ milk at 2 years of age. Parents will also be provided bi-monthly email reminders. Children who receive the whole milk recommendation will be provided with the same age-appropriate nutritional recommendations for foods other than cow's milk as children who receive the reduced fat recommendation as part of routine healthcare according to the Rourke Baby Record. ${ }^{31}$

\section{Reduced fat milk recommendation}

Children randomised to the reduced fat group will receive a primary care recommendation to transition from whole milk to $500 \mathrm{~mL}$ of reduced fat (1\%) milk per day once the child is 2 years of age (consistent with current guidelines). Parents will also be provided bi-monthly email reminders. Children who receive the reduced fat recommendation will be provided with the same age-appropriate nutritional recommendations for foods other than cow's milk as children who receive the whole fat recommendation as part of routine healthcare according to the Rourke Baby Record. $^{31}$

\section{Adherence}

Multiple methods will be used to maximise adherence to milk recommendations: (1) primary healthcare providers will be reminded to repeat milk recommendations at up to two subsequent well-child visits during study participation; (2) research assistants will provide participants with reminder magnets specific to their allocation group (see online supplementary file) after receiving milk fat recommendations from the physician and (3) participants will receive an email survey bi-monthly which will ask about the enrolled child's recent milk consumption and remind families of the milk fat recommendation provided to them (see online supplementary file 1 for email script). 


\section{Baseline participant characteristics}

The following baseline variables will be measured: age, sex, zBMI, z-height, birth weight, waist circumference, ethnicity, maternal age and education level, duration of breast feeding, current and past vitamin D supplementation, daily volume of cow's milk intake, daily multivitamin use, parental BMI, screen viewing time, physical activity, sleep time and total dietary intake in the past 24 hours using the standardised TARGet Kids! data collection instrument adapted from the Canadian Community Health Survey $^{32}$ : cognitive development will be measured using the ASQ. ${ }^{33}$

\section{Follow-up outcome measures}

Follow-up of parents and children will occur at 24 months postrandomisation, which has been used in previous childhood obesity prevention trials. ${ }^{34}{ }^{35}$ Follow-up will be completed by trained research assistants at each practice site during routine healthcare using the same techniques as baseline.

\section{Primary outcome}

The primary outcome will adiposity, measured by the mean difference in age-standardised and sexstandardised zBMI which will be measured at 24 months postrandomisation. zBMI is an important outcome that is predictive of adiposity in later childhood, adolescence and adulthood. ${ }^{36}{ }^{37}$ Using standardised anthropometric protocols, ${ }^{38}{ }^{39}$ trained research assistants will measure children's height using a Healthometer Stadiometer (Healthometer, Boca Raton, Florida, USA) and weight will be measured using a digital Healthometer Scale (Healthometer). BMI will be calculated as weight $(\mathrm{kg})$ divided by height $\left(\mathrm{m}^{2}\right)$. zWC will also be used to assess adiposity, and will be measured by trained research assistance according to standardised anthropometric protocols. ${ }^{38}{ }^{39}$ Waist circumference has been associated with future weight gain, diabetes and cardiovascular disease and is the recommended measure for children with obesity. ${ }^{40-43}$ Both BMI and WC will be standardised using the WHO growth references ranges (zBMI and zWC) ${ }^{44} 45$ which reflect optimal childhood growth and are recommended for clinical use in Canada. ${ }^{46}$ Velocity of zBMI change (from baseline to trial termination), which is predictive of higher BMI in adolescence and adulthood, will be used to measure differences in growth rate. ${ }^{3747}$

\section{Secondary outcomes \\ Cardiovascular health}

Laboratory measures including glucose, hsCRP, nonHDL, LDL, triglyceride, HDL and total cholesterol, insulin and diastolic and systolic blood pressure will be obtained since these measures track from childhood to adulthood and are important early indicators of cardiovascular health. ${ }^{48-51}$ Non-fasting blood samples will be taken during the clinic visit by TARGet Kids! research assistants who are trained phlebotomists. Non-fasting measures have been established as equivalent to fasting measures, which are not feasible from young children. ${ }^{52}$ Existing paediatric reference standards will be used to identify high risk children or the 90th percentile when these are unavailable. ${ }^{53}$

\section{Vitamin D}

Vitamin D will be measured by serum $25(\mathrm{OH}) \mathrm{D}$ concentration from venous blood at baseline and follow-up using isotope dilution liquid chromatography tandem mass spectrometry ${ }^{54}$ by the Mount Sinai Services (MSS) Laboratory (mountsinaiservices.ca). ${ }^{55}$

\section{Child cognitive development}

Child cognitive development will be assessed by parental report using the $\mathrm{ASQ}^{33}$ at enrolment and follow-up visits. The ASQ is a parent completed developmental questionnaire which has been cross-culturally validated and is routinely used during primary healthcare as recommended by the American Academy of Pediatrics. It identifies children at risk of developmental delay across five domains: communication, gross motor, fine motor, problem solving and personal social behaviour. In addition, the junior and senior kindergarten teacher completed EDI will be collected in both junior and senior kindergarten to assess overall child development and school readiness. The EDI is collected across Canada for population-level monitoring of child development and covers five developmental domains: physical health and well-being, social competence, emotional maturity, language and cognitive development, communication skills and general knowledge. ${ }^{56-58}$

\section{Sugary beverage and total energy intake}

The Automated Self-Administered 24 hours Assessment (ASA24) from the National Cancer Institute of the National Institutes of Health will be used to measure sugary beverage and total energy intake at baseline and follow-up. ${ }^{59}$ Differences in fat intake from both cow's milk and other dietary sources of fat will be evaluated. The ASA24 is a web-based tool that allows 24 hours food recall modelled after the United States Department of Agriculture (USDA) Automated Multiple-Pass Method. The ASA24 has been validated for sugary beverage and total energy intake and has been piloted in Ontario children aged 2-5 years for feasibility of completion within 30 min. $^{59-61}$

\section{Sample size}

Previous obesity prevention trials have established a clinically meaningful zBMI difference of $\geq 0.25{ }^{6263}$ To detect this difference, 426 children will be required in each group ( $\mathrm{n}=213$ per group), based on an alpha of 0.05 with $80 \%$ power. To accommodate $20 \%$ loss to follow-up, ${ }^{64} 534$ $1.5-2.99$ years old children will be recruited $(n=267$ per group) over 2 years and subsequently followed for 2 years.

\section{Recruitment}

Recruitment strategies include recruitment in person by a trained research assistant who is known to them through 
the TARGet Kids! programme at a routine primary healthcare visit and collecting non-invasive measures (questionnaires and anthropometric measures) while the child and family wait for their primary healthcare provider visit.

\section{Randomisation}

Children will be randomised using a 1:1 allocation ratio to either group. Randomisation will be computer generated with variable block sizes, and will be stratified by site to ensure a balanced distribution of participants between groups within each of the sites. ${ }^{65}$ Web-based central randomisation will be used to preserve allocation concealment. After a child has been determined eligible to participate in CoMFORT and parents provide informed consent, research assistants will access the online central randomisation system to ascertain the child's randomisation status.

\section{Blinding}

Due to the nature of the study, children and parents cannot be blind, but they will be blind to the trial hypotheses. Primary healthcare providers who provide the recommendations also cannot be blind. Allocation concealment will be preserved for research assistants and parents who may enrol participants depending on randomisation sequence if they are aware of it.

\section{Retention and complete collection of data}

Every reasonable attempt (including phone calls and emails) will be made to locate CoMFORT subjects at follow-up. All families will be reminded via phone call to attend their scheduled annual well-child visit, consistent with routine practice. To further reduce loss to follow-up, parents who have moved out of district will be offered to visit The Hospital for Sick Children for repeat laboratory, cognitive and anthropometric testing.

\section{Data management}

The Applied Health Research Centre of the Li Ka Shing Knowledge Institute of St. Michael's Hospital and Peter Gilgan Centre for Research and Learning of The Hospital for Sick Children will be the data coordinating centres. Study data were collected and managed using Research Electronic Data Capture (REDCap) electronic data capture tools hosted at St. Michael's Hospital. ${ }^{66}$ REDCap is a secure, web-based application designed to support data capture for research studies, providing: (1) an intuitive interface for validated data entry; (2) audit trails for tracking data manipulation and export procedures; (3) automated export procedures for seamless data downloads to common statistical packages and (4) procedures for importing data from external sources. Laboratory tests from the MSS Laboratory will be directly uploaded through a secure web portal.

\section{Statistical analysis}

Descriptive statistics for baseline characteristics (frequencies and proportions for discrete variables; means and SDs for symmetric variables; medians and IQRs for skewed data) will be used to evaluate randomisation completeness. The intent-to-treat principle will be applied to the analysis of outcomes. ${ }^{6768}$ Although randomisation is expected to balance the covariates, variables that demonstrate, by chance, a potentially clinically meaningful imbalance, will be considered as adjusting covariates. For the primary analysis, zBMI at 24 months postrandomisation will be compared between groups using ANCOVA adjusting for baseline zBMI. Because zBMI is age standardised, minimal differences in age at follow-up will be accounted for. For the secondary analyses, group differences in cognitive developmental scores, serum $25(\mathrm{OH}) \mathrm{D}$ concentration, cardiometabolic factors, sugary beverage and total energy intake will be compared using linear regression. Piecewise linear mixed models will be used to determine differences in growth rates between groups.

\section{Cost effectiveness}

An economic analysis will be conducted to determine the incremental costs (or cost-savings) of whole versus reduced fat cow's milk in reducing childhood adiposity, from both health system and societal perspectives. The time horizon will be limited to 5 years to leverage patient level data and minimise uncertainty from modelling a longer time horizon. All costs, parameter estimates and ranges will be derived from study data and will be obtained using medical record extraction. Publicly available Ontario costing sources will be used to cost resource utilisation parameters. Cost-effectiveness will be expressed as the incremental cost-effectiveness ratio (ICER), calculated by dividing the incremental costs between the intervention arms by the incremental change in child's zBMI between baseline and the end of the follow-up period. Costs will be adjusted for inflation using the Canadian Consumer Price Index and reported in 2022 Canadian dollars. An extensive one-way deterministic sensitivity analysis will be performed to evaluate the robustness of the results and evaluate uncertainty in any of the assumptions. Ranges for the sensitivity analysis will be obtained from $95 \%$ CIs generated from study data for each of the parameters. Probabilistic sensitivity analysis using Monte Carlo simulation will be used to further evaluate uncertainty and establish a point estimate and 95\% CI around the ICER. Economic evaluation analyses will be carried out through the Ontario Child Health SPOR SUPPORT Unit.

\section{Ethics and dissemination}

Approval has been obtained for the CoMFORT trial from the Unity Health Toronto (REB\# 18-369) and Hospital for Sick Children (REB\# 1000063023) Research Ethics Boards. Findings will be disseminated directly to primary healthcare providers and to parents. A meeting of all the TARGet Kids! practices, research team and policy leaders (representatives from the University of Toronto Section of Community Paediatrics, Family and Community Medicine, Ontario Medical Association, Maternal, Infant, Child and Youth Research Network, College of Family Physicians, Canadian Paediatric Society and parent 
representatives) and public health agencies (Public Health Ontario and Public Health Agency of Canada) will occur annually. Downstream dissemination to primary healthcare providers will occur through formal and informal avenues at local levels, such as City Wide Paediatric Rounds, national Continuing Medical Education events and those held by local physician groups. Both parent and clinician members of the parent panel will play an integral role in communicating trial evidence by participating in the development of all dissemination material. End of grant knowledge will be shared with the academic community through multiple publications in a high impact journal as well as presentations at national and international conferences.

\section{Consent}

A stepwise proportionate consent model will be used for this study ${ }^{69}$ First, during the TARGet Kids! cohort study consent process, participants consent to be approached for additional research. As the second step, participants who consent to TARGet Kids! and are eligible for CoMFORT will be approached to participate in CoMFORT by research assistants at either the 18-month or 2-year well-child visit. Proportionate consent will be sought according to the National Health Service (UK) proportionate consent guidelines, ${ }^{69}$ which have four components: providing a (1) verbal description of the study, (2) information sheet to participants (3) answering participant questions and (4) documenting informed consent. ${ }^{69}$ TARGet Kids! research assistants will verbally provide information to each eligible family about the nature and purpose of the research, in addition to the material risks, benefits and alternatives, and provide an information sheet about the CoMFORT trial. Informed, written consent will then be obtained before randomisation. A copy of the CoMFORT consent form can be found in the online supplementary file.

\section{Patient and public involvement}

Parents and clinicians identified this research question as important and relevant in a qualitative study using interviews and online questionnaires. ${ }^{27}$ A TARGet Kids! parent panel informed all aspects of this protocol to maximally meet the needs of parents and children, including revising patient-facing materials about the intervention and consent forms and guiding the design of the recruitment process. Parents verified the intervention as designed was appropriate and feasible. Over the course of this trial, the parent panel will meet virtually as needed and in person at least once every 6 months to discuss recruitment, study promotion and overall progress. Parents' experiences will be valued as evidence and an integral part of the research process. Parent partners will contribute to knowledge translation strategies, coauthor study publications and attend conferences alongside investigators to present study findings.

\section{IMPACT}

Results from this trial will be applicable to practice and nutrition guidelines because: (1) two widely accepted, clinically relevant alternatives will be directly compared; (2) a diverse sample of healthy children participating in routine healthcare will be involved; (3) practice settings representing the range of primary healthcare practice will be included (family medicine, primary care paediatrics, community health centres, etc); (4) patientimportant health outcomes will be measured including adiposity, child cognitive development and nutrition and (5) the multidisciplinary team includes clinicians, parents and policymakers as partners in the research process. The CoMFORT trial has been created through meaningful collaboration with parents through governance, conducting research and knowledge translation. In doing so, the results of the CoMFORT trial will be well positioned for implementation and integration into the lives of children and families.

\section{Author affiliations}

${ }^{1}$ Nutritional Sciences, University of Toronto, Toronto, Ontario, Canada

${ }^{2}$ Pediatrics, St. Michael's Hospital, Toronto, Ontario, Canada

${ }^{3}$ Li Ka Shing Knowledge Institute, St. Michael's Hospital, Toronto, Ontario, Canada

${ }^{4}$ Child Health Evaluative Sciences, The Hospital for Sick Children, Toronto, Ontario, Canada

${ }^{5}$ University of Toronto Institute of Health Policy Management and Evaluation, Toronto, Ontario, Canada

${ }^{6}$ Pediatrics, The Hospital for Sick Children, Toronto, Ontario, Canada

${ }^{7}$ McGill University, Montreal, Quebec, Canada

${ }^{8}$ Department of Pediatrics, Mcgill University, Montreal, Quebec, Canada

${ }^{9}$ Canadian Paediatric Society, Ottawa, Ontario, Canada

${ }^{10}$ University of Alberta, Edmonton, Alberta, Canada

${ }^{11}$ Offord Centre for Child Studies, McMaster University, Hamilton, Ontario, Canada

${ }^{12}$ Pediatrics, The University of British Columbia, Vancouver, British Columbia, Canada

${ }^{13}$ Health Promotion, Chronic Disease and Injury Prevention, Public Health Ontario, Ottawa, Ontario, Canada

${ }^{14}$ Clinical Trials Unit, The Hospital for Sick Children, University of Toronto, Toronto, Ontario, Canada

${ }^{15}$ Family and Community Medicine, St. Michael's Hospital, Toronto, Ontario, Canada

${ }^{16}$ University of Sheffield, Sheffield, UK

${ }^{17}$ Ontario Medical Association, Toronto, Ontario, Canada

${ }^{18}$ Patient Partner, Toronto, Ontario, Canada

Acknowledgements We thank SW, ET and all parent partners for their support, expertise and contributions to this protocol. We thank all of the participating families for their time and involvement in TARGet Kids! and are grateful to all practitioners who are currently involved in the TARGet Kids! practice-based research network.

Contributors SMV and JLM conceptualised and designed the research study, drafted the manuscript and approved the final manuscript as submitted. CB, KT, ML, DLO, PL, JAO, PW, EC, MJ, MEM, MF, AJ, GDCB, AL, PJ, M-AD, HM, HY, SW, ET, NP and $C R$ assisted in refining the study design, reviewed and revised the manuscript and approved the final manuscript as submitted.

Funding Funding was provided by the Canadian Institutes of Health Research (CIHR) Strategy for Patient Oriented Research Innovative Clinical Trial Multi-Year Grant.

Disclaimer The funding agency had no role in study design; in the writing of the report and in the decision to submit the article for publication.

Competing interests JLM received an unrestricted research grant for a completed investigator-initiated study from the Dairy Farmers of Canada (2011-2012) and Ddrops provided non-financial support (vitamin D supplements) for an investigator initiated study on vitamin $D$ and respiratory tract infections (2011-2015). 
Patient and public involvement Patients and/or the public were involved in the design, or conduct, or reporting, or dissemination plans of this research. Refer to the Methods section for further details.

\section{Patient consent for publication Not required}

Provenance and peer review Not commissioned; externally peer reviewed.

Open access This is an open access article distributed in accordance with the Creative Commons Attribution Non Commercial (CC BY-NC 4.0) license, which permits others to distribute, remix, adapt, build upon this work non-commercially, and license their derivative works on different terms, provided the original work is properly cited, appropriate credit is given, any changes made indicated, and the use is non-commercial. See: http://creativecommons.org/licenses/by-nc/4.0/.

ORCID iDs

Magdalena Janus http://orcid.org/0000-0002-9500-6776

Nav Persaud http://orcid.org/0000-0003-3327-5580

Jessica A Omand http://orcid.org/0000-0003-2095-3629

Jonathon L Maguire http://orcid.org/0000-0002-4083-8612

\section{REFERENCES}

1 Fulgoni VL, Quann EE. National trends in beverage consumption in children from birth to 5 years: analysis of NHANES across three decades. Nutr J 2012;11:92.

2 Garriguet D. Beverage consumption of children and teens, 2008.

3 Service NH. What to feed young children. NHS choices 2016, 2018. Available: https://www.nhs.uk/conditions/pregnancy-and-baby/ understanding-food-groups/\#milk-and-dairy-products

4 Committee NaGCPEA. Feeding your baby in the first year. Caring for Kids, 2014. Available: http://www.caringforkids.cps.ca/handouts/ feeding_your_baby_in_the_first_year[Accessed 6 Oct 2015].

5 American Academy of Pediatrics. Nutrition tools, 2011.

6 National Institutes of Health. Age-appropriate diet for children. Medline Plus 2011

7 Health Canada. Eating well with Canada's food guide, 2011.

8 Guyatt GH, Oxman AD, Vist GE, et al. Grade: an emerging consensus on rating quality of evidence and strength of recommendations. BMJ 2008;336:924-6.

9 Allen RE, Myers AL. Nutrition in toddlers. Am Fam Physician 2006;74:1527-32.

10 Physicians; AAoF. SORT: the Strength-of-Recommendation taxonomy. Available: http://www.aafp.org/dam/AAFP/documents/ journals/afp/sortdef07.pdf

11 Vanderhout SM, Birken CS, Parkin PC, et al. Relation between milkfat percentage, vitamin D, and BMI Z score in early childhood. Am J Clin Nutr 2016;104:1657-64.

12 Huh SY, Rifas-Shiman SL, Rich-Edwards JW, et al. Prospective association between milk intake and adiposity in preschool-aged children. J Am Diet Assoc 2010;110:563-70.

13 Scharf RJ, Demmer RT, DeBoer MD. Longitudinal evaluation of milk type consumed and weight status in preschoolers. Arch Dis Child 2013;98:335-40.

14 Vanderhout SM, Aglipay M, Torabi N, et al. Whole milk compared with reduced-fat milk and childhood overweight: a systematic review and meta-analysis. Am J Clin Nutr 2020;111:266-279.

15 Montmayeur J-P, Le Coutre J. Fat detection: taste, texture, and post ingestive effects. CRC Press, 2009.

16 Rolland-Cachera MF, Akrout M, Péneau S. Nutrient intakes in early life and risk of obesity. Int J Environ Res Public Health 2016;13. doi:10.3390/ijerph13060564. [Epub ahead of print: 06 Jun 2016]

17 Rolland-Cachera MF, Maillot M, Deheeger M, et al. Association of nutrition in early life with body fat and serum leptin at adult age. Int $J$ Obes 2013;37:1116-22.

18 Mozaffarian D, Cao H, King IB, et al. Trans-palmitoleic acid, metabolic risk factors, and new-onset diabetes in U.S. adults: a cohort study. Ann Intern Med 2010;153:790-9.

19 Racine NM, Watras AC, Carrel AL, et al. Effect of conjugated linoleic acid on body fat accretion in overweight or obese children. Am J Clin Nutr 2010;91:1157-64.

20 Kim LP, Mallo N. Maternal perceptions of Self-Weight and child weight may influence milk choice of participants in the special supplemental nutrition program for women, infants, and children (WIC). J Obes 2019;2019:1-9.

$21 \mathrm{Au} \mathrm{N}$. The health care cost implications of overweight and obesity during childhood. Health Serv Res 2012;47:655-76.

22 Chang C-Y, Ke D-S, Chen J-Y. Essential fatty acids and human brain. Acta Neurol Taiwan 2009;18:231-41.
23 Månsson HL. Fatty acids in bovine milk fat. Food Nutr Res 2008;52. [Epub ahead of print: 11 Jun 2008].

24 Jamieson EC, Farquharson J, Logan RW, et al. Infant cerebellar gray and white matter fatty acids in relation to age and diet. Lipids 1999;34:1065-71.

25 Michaelsen KF, Hoppe C, Lauritzen L, et al. Whole cow's milk: why, what and when? Nestle Nutr Workshop Ser Pediatr Program 2007;60:201-16.

26 Weiser MJ, Butt CM, Mohajeri MH. Docosahexaenoic acid and cognition throughout the lifespan. Nutrients 2016;8:99.

27 Vanderhout SM, Juando-Prats C, Birken CS, et al. A qualitative study to understand parent and physician perspectives about cow's milk fat for children. Public Health Nutr 2019;22:1-8.

28 Chan A-W, Tetzlaff JM, Altman DG, et al. Spirit 2013 statement: defining standard protocol items for clinical trials. Ann Intern Med 2013;158:200-7.

29 Altman DG, Schulz KF, Moher D, et al. The revised consort statement for reporting randomized trials: explanation and elaboration. Ann Intern Med 2001;134:663-94.

30 Carsley S, Borkhoff CM, Maguire JL, et al. Cohort profile: the applied Research Group for kids (target kids!). Int J Epidemiol 2015;44:776-88.

31 Leduc D. The rourke baby record. Paediatr Child Health 1998;3:300.

32 StatsCan. Canadian community health survey, 2004.

33 Squires J, Bricker D, Potter L. Revision of a parent-completed development screening tool: ages and stages questionnaires. $J$ Pediatr Psychol 1997;22:313-28.

34 James J, Thomas P, Kerr D. Preventing childhood obesity: two year follow-up results from the Christchurch obesity prevention programme in schools (CHOPPS). BMJ 2007;335:762.

35 Taylor RW, McAuley KA, Barbezat W, et al. Two-Year follow-up of an obesity prevention initiative in children: the apple project. Am J Clin Nutr 2008;88:1371-7.

36 Rolland-Cachera MF, Deheeger M, Guilloud-Bataille M, et al. Tracking the development of adiposity from one month of age to adulthood. Ann Hum Biol 1987;14:219-29.

37 Péneau S, Giudici KV, Gusto G, et al. Growth trajectories of body mass index during childhood: associated factors and health outcome at adulthood. J Pediatr 2017; 186:e61:64-71

38 CFDCA P. Third National health and nutrition examination (NHANES III): anthropometric procedures video, 2003.

39 De Onis M, Garza C, Victora C. The who multicentre growth reference study: strategy for developing a new international growth reference. paper presented at: forum of nutrition, 2003.

40 Cook S, Weitzman M, Auinger P, et al. Prevalence of a metabolic syndrome phenotype in adolescents: findings from the third National health and nutrition examination survey, 1988-1994. Arch Pediatr Adolesc Med 2003;157:821-7.

41 Bassali R, Waller JL, Gower B, et al. Utility of waist circumference percentile for risk evaluation in obese children. Int $J$ Pediatr Obes 2010;5:97-101.

42 Maffeis C, Grezzani A, Pietrobelli A, et al. Does waist circumference predict fat gain in children? Int $J$ Obes Relat Metab Disord 2001;25:978-83.

43 Sharma AK, Metzger DL, Daymont C, et al. LMS tables for waistcircumference and waist-height ratio Z-scores in children aged 5-19 $Y$ in NHANES III: association with cardio-metabolic risks. Pediatr Res 2015;78:723-9.

44 World Health Organization. Interpreting growth indicators. training course on child growth assessment, 2008. Available: http://www. who.int/childgrowth/training/module_c_interpreting_indicators.pdf [Accessed 7 July 2015].

45 Organization WH. Child growth standards. BMI-for-age. Geneva, 2006.

46 Shields M, Tremblay MS. Canadian childhood obesity estimates based on who, IOTF and CDC cut-points. Int J Pediatr Obes 2010;5:265-73.

47 Giudici KV, Rolland-Cachera M-F, Gusto G, et al. Body mass index growth trajectories associated with the different parameters of the metabolic syndrome at adulthood. Int J Obes 2017;41:1518-25.

48 Sun SS, Grave GD, Siervogel RM, et al. Systolic blood pressure in childhood predicts hypertension and metabolic syndrome later in life. Pediatrics 2007;119:237-46.

49 Chen X, Wang Y. Tracking of blood pressure from childhood to adulthood. Circulation 2008;117:3171-80.

50 Webber LS, Srinivasan SR, Wattigney WA, et al. Tracking of serum lipids and lipoproteins from childhood to adulthood. The Bogalusa heart study. Am J Epidemiol 1991;133:884-99.

51 Morrison JA, Glueck CJ, Horn PS, et al. Pre-teen insulin resistance predicts weight gain, impaired fasting glucose, and type 2 diabetes at age 18-19 Y: a 10-y prospective study of black and white girls. Am J Clin Nutr 2008;88:778-88. 
52 Birken CS, Lebovic G, Anderson LN, et al. Association between vitamin $\mathrm{D}$ and circulating lipids in early childhood. PLoS One 2015;10:e0131938.

53 Expert Panel on Integrated Guidelines for Cardiovascular Health and Risk Reduction in Children and Adolescents, National Heart, Lung, and Blood Institute. Expert panel on integrated guidelines for cardiovascular health and risk reduction in children and adolescents: summary report. Pediatrics 2011;128:S213-56.

54 Maunsell Z, Wright DJ, Rainbow SJ. Routine isotope-dilution liquid chromatography-tandem mass spectrometry assay for simultaneous measurement of the 25-hydroxy metabolites of vitamins D2 and D3. Clin Chem 2005;51:1683-90.

55 Carter GD, Carter R, Jones J, et al. How accurate are assays for 25-hydroxyvitamin D? data from the International vitamin D external quality assessment scheme. Clin Chem 2004;50:2195-7.

56 Janus M, Offord DR. Development and psychometric properties of the early development instrument (EDI): a measure of children's school readiness. Can J Behav Sci 2007;39:1-22.

57 Santos R, Brownell M, Ekuma O, et al. The early development instrument (EDI) in Manitoba, 2012.

58 Pearce A, Scalzi D, Lynch J, et al. Do thin, overweight and obese children have poorer development than their healthy-weight Peers at the start of school? findings from a South Australian data linkage study. Early Child Res Q 2016;35:85-94.

59 Subar AF, Kirkpatrick SI, Mittl B, et al. The automated selfadministered 24-hour dietary recall (ASA24): a resource for researchers, clinicians, and educators from the National cancer Institute. J Acad Nutr Diet 2012;112:1134-7.
60 Pavarin-De Luca AK, Forbes S, Haines J. A validation study for preschool aged children using parental proxy reporting. University of Guelph, 2017.

61 Moshfegh AJ, Rhodes DG, Baer DJ, et al. The US department of agriculture automated Multiple-Pass method reduces bias in the collection of energy intakes. Am J Clin Nutr 2008;88:324-32.

62 Ford AL, Hunt LP, Cooper A, et al. What reduction in BMI SDS is required in obese adolescents to improve body composition and cardiometabolic health? Arch Dis Child 2010;95:256-61.

63 Reinehr T, Andler W. Changes in the atherogenic risk factor profile according to degree of weight loss. Arch Dis Child 2004;89:419-22.

64 Aglipay M, Birken CS, Parkin PC, et al. Effect of high-dose vs standard-dose Wintertime vitamin D supplementation on viral upper respiratory tract infections in young healthy children. JAMA 2017;318:245-54.

65 Chan A-W, Tetzlaff JM, Gøtzsche PC, et al. Spirit 2013 explanation and elaboration: guidance for protocols of clinical trials. BMJ 2013;346:e7586

66 Harris PA, Taylor R, Thielke R, et al. Research electronic data capture (REDCap)--a metadata-driven methodology and workflow process for providing translational research informatics support. J Biomed Inform 2009;42:377-81.

67 Fisher LD, Herson DO, Frankowski J, et al. Intention to treat in clinical trials. statistical issues in drug research and development. New York: Marcel Dekker, 1990.

68 Maguire JL, Birken CS, Loeb MB, et al. DO IT Trial: vitamin D Outcomes and Interventions in Toddlers - a TARGet Kids! randomized controlled trial. BMC Pediatr 2014;14:37.

69 National Health Service HRA. Applying a proportionate approach to the process of seeking consent, 2017. 